López, Serrano, Gómez y García (2012) / Revista de Empresa Familiar, 2(2), 7-21.

www.revistadeempresafamiliar.uma.es

\title{
El efecto del familiness en la performance innovadora de las empresas familiares: un análisis exploratorio
}

\section{An exploratory study of familiness: Effects on innovative performance in the family firm}

\author{
María Concepción López Fernández ${ }^{\mathrm{a},{ }^{*}}$ - Ana María Serrano Bedia ${ }^{\mathrm{b}} \cdot$ Raquel Gómez López $^{\mathrm{c}}$. \\ Gema García Piqueres ${ }^{\mathrm{d}}$ \\ ${ }^{a, b, c, d}$ Departamento de Administración de Empresas, Universidad de Cantabria, Av. Los Castros s/n, 39005 \\ Santander (Spain)
}

D A T OS A R TÍ C U L O

Historial:

Recibido 15-09-2012

Aceptado 30-10-2012

Palabras clave:

Familiness

Performance

Innovación

Empresa Familiar

Códigos JEL:

L25, L26, M10

\section{A R T I C LE IN F O}

Article history:

Received 15-09-2012

Accepted 30-10-2012

Keywords:

Familiness

Performance

Innovation

Family Business

JEL codes:

L25, L26, M10

\begin{abstract}
RES UMEN
Una de las cuestiones que las investigaciones sobre empresa familiar se esfuerzan en conocer es la influencia que tiene la familia en la empresa. Generalmente se estudia su efecto sobre las distintas prácticas empresariales. Sin embargo, cuando los investigadores valoren el rendimiento de estas organizaciones deben emplear indicadores que recojan el carácter familiar de estas firmas. Además, se deben tener en consideración las implicaciones derivadas del marco teórico empleado, como puede ser la teoría de la agencia, la de recursos y capacidades u otras. Por ello, tras realizar una revisión del estado de la cuestión, se propone un indicador que recoja todas estas particularidades y sea útil para la medida del rendimiento en estas organizaciones. Finalmente, la escala elaborada se valida aplicándose sobre una muestra de empresas familiares.
\end{abstract}

\section{A B S T R A C T}

One of the issues that family business research strives to know is the influence of the family in the business practices. When researchers assess the performance of these organizations should use indicators to collect the family character of these firms. On the other hand, we must consider the implications of the theoretical framework employed, such as the agency theory or the resource based view. After a review of the state of affairs, this paper proposes an indicator that includes all these features and is useful for measuring performance in these organizations. Finally, the proposed scale is validated on a sample of family businesses.

\footnotetext{
* Autor de contacto.

Correoselectrónicos:lopezm@unican.es,serranoa@unican.es,gomezlr@unican.es,garciapg@unican.es
} 


\section{Introducción}

La importancia económica y social de las empresas familiares ha despertado el interés de académicos e investigadores y así, desde sus inicios en la década de los 60 , la investigación en empresa familiar ha alcanzado un desarrollo que le ha permitido irse abriendo camino como un campo de estudio riguroso e independiente (Astrachan et. al, 2002). No obstante, todavía se considera a la disciplina en estado emergente (Benavides et al., 2011), como lo demuestra la existencia de una serie de vacíos en la investigación, entre los que se incluye el estudio de la innovación (Zahra y Sharma, 2004; Casillas y Acedo, 2007).

En la economía moderna la innovación es considerada como un factor clave, por cuanto en un entorno de competencia creciente la competitividad de las empresas y de la economía en su conjunto va a depender en gran medida de su capacidad de innovación (Porter, 1990). Sin embargo, y a pesar de los proclamados beneficios de la relación entre innovación y performance de la empresa, esto no ocurre así en todos los casos (Capon et al., 1990; Hart, 1992; Li y Atuahene-Gima, 2001; De Clercq et al., 2009). La evidencia empírica contradictoria respecto a si la innovación redunda o no en mayor rendimiento sugiere que la obtención de beneficios en este sentido puede depender del tipo de estrategia de innovación perseguida por cada empresa, así como de la forma en que la misma es implementada.

Más concretamente, los estudios que han abordado la relación entre empresas familiares e innovación son bastante escasos en la literatura académica (Craig y Moores, 2006; Craig y Dibrell, 2006; Chen y Hsu, 2009; Wagner, 2010). A pesar de esta limitada investigación, pueden encontrarse argumentos opuestos en la literatura acerca del papel que desempeña la innovación en las empresas familiares. Así, algunos autores sugieren que las empresas familiares pueden ser muy innovadoras y agresivas en sus mercados (Aronoff, 1998) y un recurso clave para la innovación tecnológica y el desarrollo económico (Zahra, 2005; Chen y Hsu, 2009), mientras otros consideran que los negocios familiares son muy conservadores y aversos al riesgo, $\mathrm{y}$ por lo tanto menos innovadores que las empresas no familiares (Donckels y Frohlich, 1991; Gallo, 1995).

En este sentido, diversos autores han puesto de manifiesto que la existencia de un conjunto de recursos y capacidades intrínsecos de la empresa familiar, denominados genéricamente "familiness" (Cabrera-Suárez et al., 2001; Chrisman et al., 2003; Kellermans y Eddleston, 2006; Cruz y Nordqvist, 2012) puede explicar las diferencias de comportamiento y resultados de las empresas familiares, si bien hasta la fecha la investigación dirigida a explorar la influencia de estas variables en la performance innovadora de este tipo de empresas es muy limitada, si no inexistente.

En base a estas consideraciones previas el estudio que aquí se plantea, de naturaleza básicamente exploratoria, tiene como objetivo identificar el efecto del familiness en la performance innovadora de las empresas familiares.

Para dar cumplimiento a este objetivo el resto del trabajo se organiza como sigue. En el siguiente apartado se hace referencia al marco teórico empleado en la investigación. El tercer apartado recoge la metodología utilizada en el estudio empírico. En el apartado 4 se presentan los principales resultados obtenidos en la investigación. Para finalizar se muestran las principales conclusiones que se derivan del estudio, así como las limitaciones y futuras líneas de investigación.

\section{Revisión de la literatura}

Habida cuenta de la importancia que los marcos teóricos presentan en la consolidación de una disciplina científica, diversos autores están enfocando sus investigaciones hacia el desarrollo de una teoría unificada de empresa familiar cuya necesidad fue puesta de manifiesto por Wortman (1994) hace más de una década.

Destaca la utilización de dos corrientes teóricas principalmente. Por un lado, la aplicación al campo de la empresa familiar de la teoría de la agencia. Esta teoría trata de explicar 
que en empresas en las que se produce una separación entre propiedad y control, el interés de los accionistas y directivos puede divergir surgiendo diferentes problemas (Jensen y Meckling, 1976). Bajo este enfoque cabe esperar que cuando la propiedad y la dirección residen dentro de una familia los costes de agencia serán más bajos, o incluso nulos, que en empresas que no cuentan con el componente familiar (Chrisman et al., 2003). Sin embargo, se han identificado factores como el altruismo o el entrenchment que pueden generar costes de agencia (Lubatkin et al., 2005; Gómez-Mejía et al., 2001).

Por otra lado, el enfoque basado en los recursos es otro de utilidad para identificar los recursos y capacidades que hacen que las Empresas Familiares sean únicas y que les permiten desarrollar ventajas competitivas provenientes del subsistema familia (Barney, 1991). Una de las primeras consecuencias de la aplicación de dicho enfoque al campo de la Empresa Familiar es la acuñación del término familiness por parte de los investigadores para definir un rasgo distintivo y único de las Empresas Familiares proveniente de los recursos internos de los que éstas disponen debido a la interacción entre la familia, los individuos integrados en la empresa y la propia empresa. (Habbershon y Williams, 1999). En esta línea se han realizado trabajos que sugieren que las Empresas Familiares tienen ventajas competitivas en la adquisición/posesión de recursos como capital humano o capital social (Haynes et al., 1999; Aldrich y Cliff, 2003; Sirmon y Hitt, 2003); que la cultura organizativa, propuesta como un recurso inimitable, afecta a las actividades de emprendimiento de las Empresas Familiares (Zahra, Hayton y Salvato, 2002); y que la transferencia del conocimiento tácito es importante para mantener o incrementar una ventaja competitiva, ya que el éxito de una Empresa Familiar suele basarse en la única experiencia del predecesor (Cabrera-Suárez et al., 2001; Steier, 2001).

Recientes desarrollos teóricos están utilizando la teoría del capital social para profundizar en el constructo familiness e identificar sus dimensiones, así como explorar cómo se produce la creación de recursos y capacidades únicas de las empresas familiares mediante la interacción de la familia y la empresa (Pearson et al., 2008; Sharma, 2008). Aunque el familiness es difícil de concretar empíricamente, sí que es cierto que las diferencias entre empresas familiares y no familiares suelen estudiarse teniendo en cuenta la implicación familiar en la dirección de la empresa (Anderson y Reeb, 2003; Villalonga y Amit, 2006). Siguiendo a Minichilli et al. (2010), dicha implicación familiar puede medirse tomando como referencia dos aspectos o cuestiones principales en la empresa familiar. Por un lado, se encuentra el hecho de que la persona que ocupa el puesto de máximo ejecutivo o CEO de la empresa sea o no un miembro de la familia. Por otro lado, la segunda cuestión hace referencia al grado de participación de los miembros de la familia en la dirección de la empresa.

En este trabajo se analizan ambos aspectos con el objeto de identificar el efecto del familiness en la performance innovadora de las empresas familiares. En primer lugar, se analiza el grado de implicación de la familia tanto en el control de la propiedad de la empresa como en la gestión de la misma (Chua et al., 1999; Zahra 2005). En esta línea, la Teoría de la Agencia destaca que a medida que aumenta la propiedad, el propietario y la empresa logra un mayor alineamiento (Fama y Jensen, 1983; Jensen y Meckling 1976). La alineación de intereses entre la empresa y la familia propietaria contribuye a la realización de actividades que llevan asociado un riesgo tales como la innovación (Zahra, 2005). Esto se debe a que las empresas con un mayor nivel de participación de la familia están bajo menos presión para obtener mayores ganancias en el corto plazo y tienen una mayor visión a largo plazo que otros tipos de empresas (Donckels y Frölich, 1991; James, 1999; Mustakallio et al., 2002; Bruton, Ahlstrom, y Wan 2003) para mejorar el crecimiento del negocio, crear oportunidades para sus hijos, y proteger a la empresa familiar de los competidores agresivos (Poza, 2004). 
En segundo lugar se analiza la antigüedad del CEO en su puesto y el nivel de estudios del mismo.

Varios estudios se han centrado en el análisis de la antigüedad del CEO y los resultados en las empresas no familiares (Walters et al. 2007). Sus resultados muestran que largos periodos del CEO en su puesto suelen disminuir su adquisición de conocimiento, crecimiento $\mathrm{y}$ desarrollo (Audia et al. 2000), su compromiso con el aprendizaje y reduce su búsqueda de la información (Finkelstein y Hambrick, 1996), lo que dificulta el rendimiento (Miller, 1990, 1993). En el caso particular de las empresas familiares el hecho de que el CEO permanezca en el puesto durante mucho tiempo guiado por el deseo de mantener el control (Muñoz-Bullón y Sánchez-Bueno, 2011) podría permitir centrarse en aquellas actividades innovadoras que pueden rejuvenecer las operaciones de sus empresas y mejorar su posición competitiva (Zahra, 2005).

Además, se propone la idea de que un CEO que lleva años en su puesto en la empresa familiar puede proporcionar un fuerte control formal e informal que mejore, entre otros aspectos, las relaciones e inversiones necesarias para llevar a cabo actividades de innovación.

Por otro lado, en la literatura de empresa familiar existen varios trabajos que han analizado el nivel de estudios del CEO (Galve y Salas, 2003; Gallo et al., 2004; Carrasco y Meroño, 2011) y cuyos resultados muestran evidencia empírica de la existencia de diferencias significativas entre empresas familiares y no familiares, como consecuencia de la formación universitaria del gerente, resultando mucho menor, esta formación en las empresas familiares. También se han detectado diferencias entre empresas familiares, identificando a la formación del gerente como causante de las diferencias entre dichas empresas. Asimismo, el nivel de formación se relaciona de forma positiva con la capacidad del directivo para realizar elecciones estratégicas de acuerdo con las exigencias del entorno (Wiersema y Bantel, 1992).

\section{Metodología}

\subsection{Fuente de datos}

Dada la naturaleza de la investigación y la consecuente inexistencia de fuentes de datos secundarias para la consecución del objetivo planteado, se ha recurrido a la realización de encuestas para obtener la información necesaria para la realización de este estudio. Así, se ha utilizado la base de datos SABI (Sistema de Análisis de Balances Ibéricos) para seleccionar la población objetivo compuesta por empresas con domicilio social en Cantabria, con forma jurídica de sociedad anónima o de sociedad limitada, con facturación anual superior a dos millones de euros y con más de 10 empleados. El número total ascendió a 858 empresas. Después de eliminar las empresas que se encontraban en concurso de acreedores y las empresas que estaban extinguidas o resultaron ilocalizables, la población a la que se dirigió la investigación constaba de 815 empresas.

El cuestionario fue enviado al CEO de las 815 empresas de la población primero por correo postal y posteriormente electrónico durante los meses de mayo y junio de 2010. El número final de empresas que contestaron correctamente el cuestionario y que componen la muestra es de 110, lo que supone una tasa de respuesta del $13,5 \%$, similar a la registrada en otros estudios de este tipo.

\section{Tabla 1}

Ficha técnica del estudio.

\begin{tabular}{ll} 
Universo & $\begin{array}{l}\text { Empresas con domicilio social en la } \\
\text { Comunidad Autónoma de Cantabria }\end{array}$ \\
\hline $\begin{array}{l}\text { Ámbito } \\
\text { geográfico } \\
\text { Tamaño muestral }\end{array}$ & $\begin{array}{l}\text { Comunidad Autónoma de Cantabria } \\
110 \text { encuestas }(13,5 \%) \\
\text { Para el conjunto de la muestra y en } \\
\text { el supuesto de muestreo aleatorio } \\
\text { simple, se establece en el 8,7\% }\end{array}$ \\
$\begin{array}{l}\text { Error muestral } \\
\text { Nivel de confianza }\end{array}$ & $\begin{array}{l}\text { Aleatorio simple } \\
\text { Trabajo muestral de campo }\end{array}$ \\
\hline
\end{tabular}

Utilizando la prueba Chi cuadrado se ha podido contrastar la no existencia de diferencias estadísticamente significativas entre la distribución de la población y la distribución de la muestra, tanto con respecto al tamaño de las 
empresas, medido según el número de empleados, como en cuanto al sector, pudiéndose concluir, por lo tanto, que la muestra utilizada es representativa de la población objetivo del trabajo con una probabilidad de error del tipo I del 1\%.

\subsection{Análisis del sesgo de no respuesta}

Asimismo, se ha utilizado la prueba $U$ de Mann-Whitney para determinar la posible presencia de sesgo de no respuesta, a fin de evaluar si existen o no diferencias estadísticamente significativas entre las empresas que han respondido al cuestionario y las que no lo han hecho. Con esta finalidad, se han examinado las diferencias entre las medias obtenidas para las variables tamaño y sector de las empresas que respondieron al primer envío del cuestionario y las medias para esas mismas variables de las últimas respuestas recibidas. $\mathrm{La}$ justificación de este análisis descansa en la asunción generalizada de que las empresas más tardías en su respuesta son más similares a la población general que las primeras, por lo que se acepta que la comparación de las respuestas recibidas por parte de ambos grupos es un método adecuado para valorar si la tasa de respuesta introduce sesgos en los análisis (Armstrong y Overton, 1977; Chen et al., 2004; Narasimhan y Das, 2001; Das y Joshi, 2007).

Tal y como se muestra en la Tabla 2, los resultados permiten concluir que no existen diferencias estadísticamente significativas entre las primeras empresas que contestaron al cuestionario y las que lo hicieron en la segunda etapa. Del mismo modo, podemos asumir que tampoco existen diferencias estadísticamente significativas entre las empresas que han contestado a la encuesta y las que no lo han hecho. Por lo anteriormente expuesto, se puede asumir la ausencia de sesgo de no respuesta.

Tabla 2

Análisis del sesgo de no respuesta.

\begin{tabular}{ccc}
\hline & $\begin{array}{c}\text { U } \\
\text { Mann-Whitney }\end{array}$ & $\begin{array}{c}\text { Sig. Asintót. } \\
\text { (bilateral) }\end{array}$ \\
\hline SECTOR & 818 & 0,306 \\
TAMAÑO & 834,5 & 0,355 \\
\hline
\end{tabular}

3.3. Distribución y caracterización de la muestra

Para poder llevar a cabo el estudio el primer paso es establecer qué se entiende por empresa familiar en este trabajo. En este sentido y siguiendo a Dyer (2006) se entiende por Empresa Familiar aquella en la que la propiedad está en manos de una familia en más del $50 \%$ y que, a su vez, algún miembro de la familia gestiona la empresa. De acuerdo a este criterio, del total de 110 empresas de la muestra se tiene que el $69 \%$ de las mismas son familiares.

Por lo que se refiere a la caracterización de la muestra, el análisis de las Empresas Familiares muestra que son principalmente empresas pequeñas, pertenecientes en mayor medida al sector servicios, y con una orientación estratégica exploradora. Además se comprueba que las Empresas Familiares tienen una escasa vocación internacional (Tabla 3).

Tabla 3

Caracterización de las Empresas Familiares de la muestra.

\begin{tabular}{lc}
\hline Empresas Familiares & \% de Empresas \\
\hline Tamaño & 72,37 \\
Pequeña & 23,68 \\
Mediana & 3,95 \\
Grande & \\
Sector & 46,05 \\
Industria & 53,95 \\
Servicios & \\
Estrategia & 48 \\
Exploradora & 16 \\
Analizadora & 29,33 \\
Defensiva & 6,67 \\
Reactiva & \\
Mercado & 39,47 \\
Local & 65,79 \\
Nacional & 26,32 \\
Internacional & \\
\end{tabular}

\subsection{Medición de las variables}

Para la realización del estudio empírico se han utilizado distintas variables clasificadas en tres grupos: variables que miden el familiness de las empresas, variables generales o de control y variables que evalúan la perfomance innovadora de las empresas. 
Respecto a las variables con las que se ha tratado de operativizar el familiness de las empresas de la muestra, tal y como se ha comentado en el apartado anterior, este trabajo analiza por un lado, el grado de implicación de la familia tanto en el control de la propiedad de la empresa como en la gestión de la misma y, por otro lado, la antigüedad del CEO en su puesto y el nivel de estudios del mismo.

Asimismo como variables generales se han tomado el tamaño, medido por el número de empleados y que clasifica a las empresas en pequeñas, medianas y grandes; el sector de actividad según sean empresas industriales o de servicios, el mercado en el que opera la empresa pudiendo ser local, nacional o internacional y la orientación estratégica de la misma. En este último caso, para medir la estrategia se ha utilizado la conocida tipología de Miles y Snow (1978) que divide a las empresas en exploradoras, analizadoras, defensivas y reactivas. La estrategia exploradora corresponde a empresas innovadoras que están dispuestas a asumir los riesgos necesarios para la prestación de nuevos productos y servicios. La estrategia analizadora es la de las empresas que no quieren ser las primeras en su sector en ofrecer un producto o servicio que no ha sido aceptado por los consumidores previamente, pero que tratan de ofrecer un producto o servicio similar que sea competitivo. En cuanto a las empresas que siguen una estrategia defensiva, son las que se apegan a lo que saben hacer y tratan de hacerlo tan bien o mejor que cualquier otra empresa. Por último, la estrategia reactiva, corresponde a empresas que optan por no seguir un plan o programa específico para ser más competitivos, sino que realizan cambios únicamente cuando se enfrentan con fuertes amenazas.

El tercer grupo está formado por diferentes medidas que tratan de recoger la performance innovadora, debido a que a pesar de la variedad de medidas utilizadas en la literatura, parece que no existe ningún indicador generalmente aceptado. En muchos casos, la elección viene determinada tanto por los objetivos de la investigación como por la disponibilidad de datos (Beneito, 2006). De hecho, parece evidente que las inconsistencias presentes en los resultados obtenidos en diferentes estudios empíricos se derivan de la dificultad por conseguir que una única medida sea capaz de capturar la complejidad de la innovación (Duchesneau et al., 1979; Souitaris, 2002). Para tratar de salvar este inconveniente, Saviotti y Metcalfe (1984) proponen que el uso de varios indicadores de la innovación puede ofrecer una mejor comprensión de la actividad de innovación, por superar el carácter incompleto de cada una de las medidas individuales.

En concreto se han tomado los siguientes indicadores. En primer lugar se analiza si, como resultado de las innovaciones llevadas a cabo por las empresas de la muestra, éstas han solicitado alguna patente. Este tipo de medida de desempeño innovador, es uno de los más utilizados en la literatura debido a que el uso de patentes presenta una serie de ventajas como la garantía de un nivel mínimo de originalidad, así como una alta probabilidad de que sea un producto innovador (Buesa et al., 2010). Por otro lado, las patentes están estrechamente relacionadas con las invenciones y cubren casi todos los campos tecnológicos (OCDE, 2004). Esta medida de desempeño innovador ha sido utilizada en diversos trabajos previos (Tabla 4).

En segundo lugar, se analiza el impacto económico de las innovaciones sobre la cifra de negocios de las empresas en 2009. En este caso la información se presenta de forma separada en función de si la innovación ha sido radical (nueva para el mercado) o incremental (nueva para la empresa). Las innovaciones radicales e incrementales representan los dos extremos de la innovación de productos (Lokshin et al., 2009). Esta distinción es relevante desde el punto de vista económico por varias razones. En primer lugar, identifica la importancia de la actividad de innovación desarrollada por la empresa en términos de mercado (Abernathy y Clark, 1985). En segundo lugar, nos permite clasificar a las innovaciones de acuerdo con su impacto en el mercado: las innovaciones incrementales representan mejoras sobre los productos existentes que no son nuevos en el mercado, mientras que las innovaciones radicales que dan lugar a la introducción de un nuevo producto en el mercado tienen un 
Descripción de las variables del estudio empírico.

\begin{tabular}{|c|c|c|}
\hline Familiness & Descripción & Estudios \\
\hline $\begin{array}{l}\text { Generación de la } \\
\text { familia con la } \\
\text { mayor parte de la } \\
\text { propiedad }\end{array}$ & $\begin{array}{l}\text { Variable que toma valor } 1 \text { si la primera generación es la que tiene mayor } \\
\text { parte de la propiedad, } 2 \text { si es la segunda, } 3 \text { si es la tercera o posterior }\end{array}$ & $\begin{array}{l}\text { Kellermanns y Eddleston (2007); Eddleston, } \\
\text { Otondo y Kellermanns (2008); Di Giuli, } \\
\text { Caselli y Gatti (2011); Kellermanns, } \\
\text { Eddleston, Sarathy y Murphy (2012) }\end{array}$ \\
\hline $\begin{array}{l}\text { Generaciones de } \\
\text { la familia } \\
\text { implicadas en la } \\
\text { dirección }\end{array}$ & $\begin{array}{l}\text { Variable que toma valor } 1 \text { si tiene una generación implicada en la } \\
\text { dirección, } 2 \text { si tiene dos generaciones y } 3 \text { si tiene tres o más } \\
\text { generaciones }\end{array}$ & $\begin{array}{l}\text { Bammens, Voordeckers y Van Gils (2008); } \\
\text { Saito (2008); Molly, Laveren y Deloof (2010) }\end{array}$ \\
\hline $\begin{array}{l}\text { Antigüedad del } \\
\text { CEO en el puesto } \\
\text { actual }\end{array}$ & $\begin{array}{l}\text { Número de años de antigüedad en el puesto del actual gerente (variable } \\
\text { numérica) clasificada por intervalos:1) } 0-10 \text { años; 2) } 11-25 \text { años; } 3 \text { ) Más } \\
\text { de } 25 \text { años }\end{array}$ & $\begin{array}{l}\text { Zahra (2005); Voordeckers, Van Gils y Van } \\
\text { den Heuvel (2007); Kellermanns, Eddleston, } \\
\text { Barnett y Pearson (2008); Tsai, Kuo, Hung } \\
\text { (2009); Minichilli, Corbetta y MacMillan } \\
\text { (2010); Li y Srinivasan (2011) }\end{array}$ \\
\hline $\begin{array}{l}\text { Máximo nivel de } \\
\text { estudios del CEO }\end{array}$ & $\begin{array}{l}\text { Variable que toma valor } 1 \text { si la formación es de Master, } 2 \text { si es titulado } \\
\text { universitario y } 3 \text { si tiene otro tipo de formación }\end{array}$ & $\begin{array}{l}\text { Adaptado de Davis y Harveston (2001); } \\
\text { Voordeckers, Van Gils y Van den Heuvel } \\
(2007)\end{array}$ \\
\hline $\begin{array}{l}\text { Variables } \\
\text { generales }\end{array}$ & Descripción & Estudios \\
\hline Tamaño & $\begin{array}{l}\text { Grande }(\geq 250 \text { empleados }) \\
\text { Mediana }(50<250 \text { empleados }) \\
\text { Pequeña( } 10<50 \text { empleados })\end{array}$ & $\begin{array}{l}\text { Clasificación de la Comisión Europea desde el } \\
1 \text { de enero de } 2005\end{array}$ \\
\hline Sector & $\begin{array}{l}\text { Industria } \\
\text { Servicios }\end{array}$ & $\begin{array}{l}\text { Clasificación Nacional de Actividades } \\
\text { Económicas } 2009 \text { (CNAE-2009) aprobada por } \\
\text { el Real Decreto 475/2007, de } 13 \text { de abril }\end{array}$ \\
\hline Mercado Local & $\begin{array}{l}\text { Internacional } \\
\text { Nacional } \\
\text { Local }\end{array}$ & \\
\hline $\begin{array}{l}\text { Orientación } \\
\text { estratégica }\end{array}$ & $\begin{array}{l}\text { Exploradora } \\
\text { Analizadora } \\
\text { Defensiva } \\
\text { Reactiva }\end{array}$ & Miles y Snow (1978) \\
\hline $\begin{array}{l}\text { Performance } \\
\text { Innovadora }\end{array}$ & Descripción & Estudios \\
\hline $\begin{array}{l}\text { Patentes } \\
\text { Impacto } \\
\text { económico } \\
\text { la cifra de } \\
\text { negocios }\end{array}$ & Variable que toma valor 1 si empresa solicitó alguna patente & Licht y Zoz (2000); Beneito (2006) \\
\hline $\begin{array}{l}\text { Innovación } \\
\text { incremental }\end{array}$ & $\begin{array}{l}\text { Porcentaje de la cifra de negocios debido a la introducción de } \\
\text { innovaciones que representan una novedad exclusivamente para la } \\
\text { empresa. }\end{array}$ & $\begin{array}{l}\text { Klomp y van Leeuwen (2001); Jantunen } \\
\text { (2005); Cassiman y Veuglers (2006); Marsili y } \\
\text { Salter, (2006); Frenz y Ietto-Gillies, (2009); } \\
\text { Kirner et al. (2009); Tsai (2009). }\end{array}$ \\
\hline Innovación radical & $\begin{array}{l}\text { Porcentaje de la cifra de negocios debido a la introducción de } \\
\text { innovaciones que representan una novedad para el mercado }\end{array}$ & \\
\hline \multirow{2}{*}{$\begin{array}{l}\text { Efectos de la } \\
\text { actividad } \\
\text { innovadora } \\
\text { Efectos externos }\end{array}$} & & \\
\hline & $\begin{array}{l}\text { Importancia que la empresa le da (likert 1-5) a cada uno de los siguientes } \\
\text { efectos del desarrollo de sus actividades de innovación: Gama más } \\
\text { amplia de bienes y servicios; Mayor calidad de los bienes y servicios; } \\
\text { Aumento o mejora de la cuota de mercado; Introducción de productos a } \\
\text { nuevos mercados o nuevos grupos de clientes; Aumento de la visibilidad } \\
\text { de los productos o negocios. }\end{array}$ & $\begin{array}{l}\text { Wheelwright y Clark, 1992; OECD- } \\
\text { EUROSTAT, 1997; Zhan y Doll, 2001; Valle y } \\
\text { Avella 2003. } \\
\text { Ahuja y Katila, 2001; Alegre y Lapiedra, 2005; } \\
\text { Alegre et al., 2006. }\end{array}$ \\
\hline Efectos internos & $\begin{array}{l}\text { Mayor flexibilidad en la producción o prestación de servicios; Mayor } \\
\text { capacidad de producción o prestación de servicios; Reducción del } \\
\text { periodo de respuesta a las necesidades de un cliente o proveedor; } \\
\text { Menores costes por unidad producida; Mejora de la satisfacción del } \\
\text { personal o disminución de la tasa de rotación del mismo; Mejora del } \\
\text { intercambio de información o comunicación dentro de la empresa }\end{array}$ & $\begin{array}{l}\text { Kusunoki et al., 1998; Alegre y Lapiedra, } \\
\text { 2005; Alegre et al., 2006. }\end{array}$ \\
\hline
\end{tabular}

impacto en dicho mercado. Esta medida de la capacidad de innovación está en consonancia con la definición de innovación, recogida en el Manual de Oslo (Caloghirou et al., 2004) y ha sido utilizado en trabajos anteriores (Tabla 4).

Finalmente se realiza un análisis de la información correspondiente al porcentaje de
Empresas Familiares que clasifican como importante o muy importante a los efectos que ha podido tener el resultado de la actividad innovadora en la empresa. Diversos autores consideran que las medidas de innovación deben abarcar aspectos más allá de los relacionados con la solicitud de patentes o el 
desarrollo de innovaciones de productos. Siguiendo este criterio, empiezan a utilizarse en la literatura un conjunto de indicadores del desempeño innovador alternativos que le clasifican en función de sus impactos. Los principales indicadores utilizados son los que hacen referencia a los efectos internos y externos de la innovación. Los efectos externos miden el grado de éxito de las innovaciones en el mercado, mientras que los internos representan el esfuerzo interno de las empresas efectuado para conseguir ese éxito (Alegre y Lapiedra, 2005). Estas dos dimensiones están en línea con trabajos previos tal y como puede verse en la Tabla 4.

En la Tabla 4 se recoge la descripción de las variables y su medición así como los estudios previos que se han llevado a cabo utilizando dichas variables.

\subsection{Análisis de los datos}

Para abordar el objetivo de este trabajo, se ha llevado a cabo un estudio de las frecuencias que facilitan las tablas de contingencia, así como el estadístico ji-cuadrado de Pearson. Este estadístico se utiliza para contrastar la hipótesis de que las dos variables categóricas analizadas son independientes. Si los datos son compatibles con la hipótesis de independencia, la probabilidad asociada al estadístico $X^{2}$ será alta $(p>0,1)$. Mientras que en caso contrario concluiremos que las variables estudiadas están relacionadas.

En concreto, se ha analizado en primer lugar el efecto que las variables generales (tamaño, sector, mercado y estrategia) tienen sobre cada uno de los indicadores de resultados: solicitud de medidas de protección de la innovación (patentes), efectos económicos $(\%$ de facturación debido a la introducción de innovaciones incrementales o radicales) e impactos no económicos con el objeto de identificar los efectos que estas variables generales pudieran tener sobre la obtención de resultados de la innovación.

En segundo lugar, se ha analizado el efecto que las variables específicas de empresa familiar (generación familiar que tiene la mayor parte de la propiedad de la empresa, miembros de la familia propietaria que ocupan puestos directivos, nivel de estudios del CEO, antigüedad del CEO, generaciones implicadas en la dirección de la empresa) tienen sobre los diferentes tipos de resultados de la innovación.

\section{Resultados}

4.1. Efecto de las variables generales sobre la performance innovadora de las Empresas Familiares.

En este apartado se analiza el efecto de las variables generales tamaño, sector, mercado y estrategia sobre los indicadores de la performance innovadora en las Empresas Familiares.

En primer lugar, se cruzaron las variables generales con el indicador patentes. Los resultados del estudio ponen de relieve la existencia de comportamientos diferentes dentro de las Empresas Familiares en cuanto a la variable estrategia. Dichos resultados sugieren un mayor nivel de solicitud de patentes por parte de las EF que siguen una estrategia exploradora (ver Tabla 5).

En segundo lugar, una vez cruzadas las variables generales con las variables que miden los impactos económicos derivados de la innovación, en concreto, el porcentaje de ventas debido a la introducción de innovaciones nuevas para el mercado (innovaciones radicales), se obtienen diferencias de comportamiento dentro de este colectivo en las variables tamaño y mercado.

Respecto del tamaño las Empresas Familiares pequeñas obtuvieron significativamente más porcentaje de ingresos de las innovaciones radicales que el resto de Empresas Familiares. Por otro lado, se observa que las Empresas Familiares que operan en mercados locales e internacionales obtuvieron significativamente más porcentaje de ingresos de las innovaciones radicales que las Empresas Familiares que operan en mercados nacionales (ver Tabla 5). 


\section{Tabla 5}

Resumen de las diferencias entre Empresas Familiares respecto a las variables generales.

\begin{tabular}{|c|c|c|c|c|c|c|c|c|}
\hline & \multirow{3}{*}{$\begin{array}{c}\text { Protección } \\
\text { de la } \\
\text { Innovación }\end{array}$} & \multicolumn{2}{|c|}{$\begin{array}{l}\text { Impacto Económico en función } \\
\text { de la innovación }\end{array}$} & \multicolumn{5}{|c|}{ Impacto cualitativo de la innovación } \\
\hline & & \multirow[b]{2}{*}{$\begin{array}{l}\text { Innovaciones } \\
\text { Radicales }\end{array}$} & \multirow[b]{2}{*}{$\begin{array}{l}\text { Innovaciones } \\
\text { Incrementales }\end{array}$} & \multirow{2}{*}{$\begin{array}{l}\text { Efectos } \\
\text { Externos } \\
\text { Aumento } \\
\text { gama de } \\
\text { bienes y } \\
\text { servicios }\end{array}$} & \multicolumn{4}{|c|}{ Efectos Internos } \\
\hline & & & & & $\begin{array}{l}\text { Comunicación } \\
\text { Interna }\end{array}$ & $\begin{array}{l}\text { Mejora de la } \\
\text { satisfacción } \\
\text { del personal }\end{array}$ & $\begin{array}{l}\text { Menores } \\
\text { costes por } \\
\text { unidad } \\
\text { producida }\end{array}$ & $\begin{array}{l}\text { Reducción } \\
\text { periodo } \\
\text { respuesta a } \\
\text { clientes o } \\
\text { proveedores }\end{array}$ \\
\hline & Sig. & Sig. & Sig. & Sig. & Sig. & Sig. & Sig. & Sig. \\
\hline Tamaño & 0,627 & 0,003 & 0,153 & 0,740 & 0,189 & 0,544 & 0,05 & 0,528 \\
\hline Sector & 0,195 & 0,418 & 0,247 & 0,282 & 0,010 & 0,031 & 0,234 & 0,117 \\
\hline Mercado & 0,618 & 0,045 & 0,107 & 0,765 & 0,660 & 0,714 & 0,720 & 0,866 \\
\hline Estrategia & 0,045 & 0,220 & 0,043 & 0,024 & 0,460 & 0,499 & 0,794 & 0,033 \\
\hline
\end{tabular}

Asimismo, respecto al impacto económico medido a través del porcentaje de ventas debido a la introducción de innovaciones nuevas para la empresa (innovaciones incrementales), se aprecian diferencias de comportamiento en cuanto a la variable estrategia, siendo las Empresas Familiares que siguen una estrategia exploradora las que obtuvieron más porcentaje de ingresos de las innovaciones incrementales (ver Tabla 5).

En tercer lugar, cuando se cruzaron las variables generales con los efectos externos, los resultados revelan la existencia de diferencias de comportamiento dentro de las Empresas Familiares para el efecto aumento de gama de bienes. En este sentido, las Empresas Familiares que siguen una estrategia defensiva son las empresas que significativamente menos valoran este tipo de efecto derivado del desarrollo de innovaciones (ver Tabla 5).

Por lo que a los efectos internos se refiere, los resultados muestran la existencia de diferencias dentro del colectivo de Empresas Familiares para la comunicación interna, la satisfacción del personal, los costes unitarios, el periodo de respuesta. Respecto de los efectos aumento de la comunicación interna y mejora de la satisfacción del personal, los resultados del estudio ponen de relieve la existencia de comportamientos diferentes dentro de las Empresas Familiares ya que las pertenecientes al sector servicios valoran significativamente más estos dos tipos de efectos de la innovación.
Continuando con el efecto de obtención de menores costes por unidad producida como consecuencia de las innovaciones, se tiene que las Empresas Familiares grandes valoran significativamente más este efecto. En cuanto al efecto reducción del periodo de respuesta a las necesidades de un cliente o proveedor son las Empresas Familiares que siguen una estrategia exploradora las que valoran significativamente más este efecto (ver Tabla 5).

\subsection{Efecto del familiness sobre los indicadores de la performance innovadora en las Empresas Familiares.}

En primer lugar se cruzaron las variables específicas de Empresa Familiar con los resultados de innovación en términos de patentes. Los resultados muestran la existencia de diferencias estadísticamente significativas únicamente en cuanto a la generación en la que se encuentra la empresa, ya que las Empresas Familiares de primera generación solicitan un número significativamente mayor de patentes que las de segunda o tercera o más (ver Tabla $6)$.

En segundo lugar, respecto a las Empresas Familiares que obtuvieron algún porcentaje de ventas debido a la introducción de innovaciones nuevas para el mercado (innovaciones radicales) o la empresa (innovaciones incrementales), sólo se encuentran diferencias significativas para el caso de las segundas en cuanto a la antigüedad 


\section{Tabla 6}

Resumen de las diferencias entre Empresas Familiares respecto a las variables específicas.

\begin{tabular}{|c|c|c|c|c|c|c|c|c|c|c|}
\hline & $\begin{array}{l}\text { Protección } \\
\text { de la }\end{array}$ & \multicolumn{2}{|c|}{$\begin{array}{l}\text { Impacto Económico en } \\
\text { función de la innovación }\end{array}$} & \multicolumn{3}{|c|}{ Efectos Externos } & \multicolumn{4}{|c|}{ Efectos Internos } \\
\hline & Patentes & $\begin{array}{l}\text { Innovaciones } \\
\text { Radicales }\end{array}$ & $\begin{array}{l}\text { Innovaciones } \\
\text { Incrementales }\end{array}$ & $\begin{array}{c}\text { Aumento } \\
\text { gama de } \\
\text { bienes }\end{array}$ & $\begin{array}{l}\text { Aumento } \\
\text { calidad } \\
\text { de } \\
\text { bienes y } \\
\text { servicios }\end{array}$ & $\begin{array}{l}\text { Aumento } \\
\text { de la } \\
\text { visibilidad }\end{array}$ & $\begin{array}{l}\text { Comunicación } \\
\text { interna }\end{array}$ & $\begin{array}{l}\text { Satisfacción } \\
\text { del personal }\end{array}$ & $\begin{array}{c}\text { Costes } \\
\text { unitarios }\end{array}$ & Flexibilidad \\
\hline & Sig. & Sig. & Sig. & Sig. & Sig. & Sig. & Sig. & Sig. & Sig. & Sig. \\
\hline $\begin{array}{l}\text { Generación } \\
\text { Propiedad }\end{array}$ & 0,007 & 0,733 & 0,655 & 0,692 & 0,006 & 0,514 & 0,243 & 0,141 & 0,209 & 0,639 \\
\hline $\begin{array}{l}\text { Generación } \\
\text { Dirección }\end{array}$ & 0,989 & 0,762 & 0,007 & 0,640 & 0,509 & 0,360 & 0,827 & 0,501 & 0,505 & 0,579 \\
\hline $\begin{array}{l}\text { Estudios } \\
\text { CEO }\end{array}$ & 0,885 & 0,257 & 0,660 & 0,036 & 0,043 & 0,044 & 0,031 & 0,012 & 0,416 & 0,012 \\
\hline $\begin{array}{l}\text { Antigüedad } \\
\text { CEO }\end{array}$ & 0,402 & 0,178 & 0,007 & 0,831 & 0,171 & 0,206 & 0,878 & 0,255 & 0,037 & 0,02 \\
\hline
\end{tabular}

del CEO y el número de generaciones implicadas en la dirección.

Los resultados apuntan que son las Empresas Familiares con antigüedad del CEO entre $11 \mathrm{y}$ 25 años y con una o dos generaciones implicadas en la dirección, las que obtienen un porcentaje de ingresos de las innovaciones incrementales significativamente mayor (ver Tabla 6).

En tercer lugar, se cruzaron las variables específicas de Empresa Familiar con los efectos externos, los resultados ponen de manifiesto la existencia de diferencias de comportamiento dentro de las Empresas Familiares para los efectos aumento de gama de bienes, aumento de la calidad de los bienes y servicios y aumento de la visibilidad. Por lo que al aumento de la gama de bienes se refiere, las Empresas Familiares con CEO Máster son las que lo valoran significativamente más. Continuando con el aumento de la calidad de bienes y servicios, este efecto es significativamente más valorado por aquellas EF en primera o tercera generación, y/o aquellas Empresas Familiares con CEO Máster. Por último, respecto del aumento de la visibilidad de los productos o negocios, los resultados indican que las Empresas Familiares con CEO Universitario valoran significativamente más este efecto de la innovación (ver Tabla 6).

Por último, y respecto a los efectos internos, los resultados muestran la existencia de diferencias para la comunicación interna, la satisfacción del personal, los costes unitarios y la flexibilidad. Respecto del aumento de la comunicación interna y mejora de la satisfacción del personal existen comportamientos diferentes dentro de las Empresas Familiares en cuanto a la formación del CEO, de manera que las empresas familiares con un CEO Universitario, valoran significativamente más estos dos tipos de efectos de la innovación.

Continuando con el efecto de obtención de menores costes por unidad producida como consecuencia de las innovaciones, se tiene que las Empresas Familiares con un CEO de antigüedad superior a 25 años lo valoran significativamente menos. En último lugar, el estudio indica que las Empresas Familiares con CEO Máster y/o una antigüedad del CEO entre 0 y 10 son las que valoran significativamente más el aumento de la flexibilidad en la producción o prestación de servicios (ver Tabla $6)$.

\section{Conclusiones}

El presente trabajo de carácter exploratorio ha tratado de cubrir el vacío existente en la literatura sobre innovación en la empresa familiar mediante el análisis del efecto del familiness en la performance innovadora de las empresas familiares. Concretamente se han utilizado las características del CEO y la implicación de la familia en la dirección y 
propiedad de la empresa como medidas del familiness de las empresas familiares de la muestra. Asimismo se han manejado tres medidas diferentes de la performance innovadora ya que según la literatura revisada parece que no existe ningún indicador generalmente aceptado.

Los análisis llevados a cabo permiten extraer conclusiones respecto los dos grupos de variables considerados.

En primer lugar, respecto a las variables generales y su efecto en la performance innovadora de las empresas familiares, cabe destacar que el hecho de estar dispuestos a asumir los riesgos necesarios para la prestación de nuevos productos y servicios, a través de una estrategia exploradora, permite obtener unos mejores resultados en términos de patentes, innovaciones incrementales y efectos internos (periodo de respuesta). Esto puede indicar un perfil innovador de las Empresas Familiares en cuanto a los objetivos que persiguen en el desarrollo de sus actividades innovadoras: no son innovadoras radicales que lideran los cambios de mercado, sino que se centran en llevar a cabo mejoras de las innovaciones ya existentes y sus actividades innovadoras surgen como respuesta a las necesidades de los clientes.

Respecto a las variables del familiness, los resultados que arroja el estudio empírico permiten concluir que el nivel de estudios o de formación del CEO afecta al impacto cualitativo de la innovación. En este sentido y habida cuenta de que el nivel de formación se relaciona de forma positiva con la capacidad del directivo para realizar elecciones estratégicas de acuerdo con las exigencias del entorno, este estudio pone de manifiesto la necesidad de desarrollar planes de formación para los propietarios y/o gestores de las empresas familiares con el objeto de ayudar al negocio a sobrevivir. Además, la profesionalización del factor humano que forma parte de la empresa dota a la misma de recursos, ideas y puntos de vista con los cuales poder construir y desarrollar la empresa adoptando algunas actividades innovadoras (Fletcher, 2002).
Por último, respecto a las variables de familiness que condicionan los resultados no cualitativos, son las empresas familiares en primera generación las que solicitan más patentes, dibujando el perfil de un fundador de carácter más claramente emprendedor. Adicionalmente, si el CEO tiene una experiencia media (entre 11 y 25 años), y hay hasta dos generaciones implicadas en la dirección, es cuando se obtienen mayores rendimientos económicos de las innovaciones incrementales realizadas. Esto confirmaría que la permanencia en el liderazgo de la empresa propio de las empresas familiares es positivo, siempre que no se lleve al extremo y que esto es compatible con la incorporación de las nuevas generaciones a las que se puede formar y de las que se pueden tomar iniciativas e ideas nuevas.

La principal limitación de este trabajo se deriva de su naturaleza exploratoria y por ello, una futura línea de investigación a seguir consiste en profundizar sobre los aspectos que han resultado significativos a la hora de analizar la performance innovadora de las empresas familiares $\mathrm{y}$, más concretamente, en la realización de estudios de carácter confirmatorio que permitan contrastar y ampliar con técnicas estadísticas más sofisticadas, los resultados que se han obtenido.

\section{Bibliografía}

Abernathy, W. J., y Clark, K. B. (1985). Innovation: mapping the winds of creative destruction. Research Policy, 14, 3-22.

Ahuja, G., y Katila, R. (2001). Technological acquisitions and the innovation performance of acquiring firms: a longitudinal study. Strategic Management Journal, 22, 197-220.

Aldrich, H. E., y Cliff, J. E. (2003). The pervasive effects of family on entrepreneurship: Towards a family embeddedness perspective. Journal of Business Venturing, 18, 573-596.

Alegre Vidal, J., y Lapiedra Alcamí, R. (2005). Gestión del conocimiento y desempeño innovador: un estudio del papel mediador del repertorio de competencias distintivas. Cuadernos de Economía y Dirección de Empresas, 23, 117-138. 
Alegre, J., Lapiedra, R., y Chiva, R. (2006). A measurement scale for product innovation performance", European Journal of Innovation Management, 9(4), 333-346.

Armstrong, J.S. y Overton, T.S. (1977). Estimating non-response bias in mail surveys. Journal of Marketing Research, 14 (3), 396-402.

Anderson, R.C., Reeb, D.M., 2003. Foundingfamily ownership and firm performance: evidence from the S\&P 500. Journal of Finance, 58, 13011328.

Aronoff, C. E. (1998). Megatrends in family business. Family Business Review, 11(3), 181-186.

Astrachan, J. H., Klein, S. B., y Smyrnios, K. X. (2002). The F- PEC scale of family influence: a proposal for solving the family business definition problem. Family Business Review, 15, 45-58.

Audia, P.G., Locke E.A., y Smith K.G. (2000). The Paradox of Success: An Archival and a Laboratory Study of Strategic Persistence Following Radical Environmental Chang. The Academy of Management Journal, 43(5), 837-853.

Bammens, Y., Voordeckers, W., y Van Gils, A. (2008). Boards of directors in family firms: a generational perspective. Small Business Economics, 31(2), 163-180.

Barney, J.B. (1991). Firm resource and sustained competitive advantage. Journal of Management, 17, 395-410.

Benavides Velasco, C.A., y Guzmán Parra, V.F. (2011) . Evolución de la literatura sobre empresa familiar como disciplina científica. CEDE, 14(2), $78-90$

Beneito, P. (2006). The innovative performance of in-house and contracted R\&D in terms of patents and utility models. Research Policy, 35(4), 502-517.

Bruton, G., Ahlstrom, D., y Wan, J.C. (2003). Turnaround in East Asian firms: evidence from ethnic Overseas Chinese communities. Strategic Management Journal, 24(6), 519-540.

Buesa, M., Heijs, J., y Baumert, T. (2010). The determinants of regional innovation in Europe: A combined factorial and regression knowledge production function approach. Research Policy, 39, 722-735.

Cabrera-Suárez, K., De Saá-Pérez, P., y GarciaAlmeida, D. (2001). The succession process from a resource- and knowledge-based view of the family firm. Family Business Review, 14, 37-46.
Caloghirou, Y., Kastelli, I., y Tsakanikas, A. (2004). Internal capability and external knowledge sources: complements or substitutes for innovative performance? Technovation, 24(1), 29-39.

Capon, N., Farley, J.U., y Hoenig, S. (1990). Determinants of financial performance: a metaanalysis. Management Science, 36, 1143-1159.

Carrasco Hernández, A. J., y Meroño Cerdan, A. L. (2011). Efectos de la formación universitaria del gerente de pymes familiares en la motivación laboral del empleado. Revista de Empresa Familiar, 1(1), 35-51.

Casillas, J., y Acedo, F. (2007). Evolution of the Intellectual Structure of Family Business Literature: a bibliometric study of FBR. Family Business Review, 20(2), 141-162.

Cassiman, B., y Veugelers, R. (2006). In Search of Complementarity in Innovation Strategy: Internal R\&D and External Knowledge Acquisition. Management Science, 5(1), 68-82.

Chen, H-L., y Hsu W-T. (2009). Family ownership, board independence, and R\&D investment. Family Business Review, 22(4), 347362.

Chen, I.J., Paulraj, A. y Lado, A.A. (2004). Strategic purchasing, supply management, and firm performance. Journal of Operations Management, $22,505-523$.

Chrisman, J. J., Chua, J. H., y Steier, L. P. (2003). An introduction to theories of family business. Journal of Business Venturing, 18, 441448.

Chua, J.H., Chrisman, J.J., y Sharma, P. (1999). Defining the family business by behavior. Entrepreneurship Theory and Practice, 23, 19-39.

Craig, J. B., y Moores, K. J. (2006). A Ten Year Investigation of Strategy, Systems and Environment Upon Innovation in Family Firm. Family Business Review, 19(1), 1-10.

Craig, J., y Dibrell, C. (2006). The natural environment, innovation, and firm performance: A comparative study. Family Business Review, 19, 275-288.

Cruz, C., y Nordqvist, M. (2012). Entrepreneurial orientation in family businesses: A generational perspective. Small Business Economics, 38, 33-49

Das, S.R. y Joshi, M.P. (2007). Process innovativeness in technology services organizations: roles of differentiation strategy, operational 
autonomy and risk-taking propensity. Journal of Operations Management, 25, 643-660.

Davis, P.S., y Harveston, P.D. (2001). The Phenomenon of Substantive Conflict in the Family Firm: A Cross-Generational Study. Journal of Small Business Management, 39(1), 4-30.

De Clercq, D., Menguc, B., y Auh, S. (2009). Unpacking the relationship between an innovation strategy and firm performance: The role of task conflict and political activity. Journal of Business Research, 62(11), 1046-1053.

Di Giuli, Caselli y Gatti (2011); Di Giuli, A., Caselli, S., y Gatti, S. (2011). Are Small Family Firms Financially Sophisticated? (February 1, 2011). Disponible en: http://ssrn.com/abstract=1016122.

Donckels, R., y Fröhlich, E. (1991). Are family businesses really different? European experiences from STRATOS. Family Business Review, 4, 149160.

Duchesneau, D., Cohn, S. F, y Dutton, J. E. (1979). A study of innovation in manufacturing: determinants, process and methodological issues. University Maine at Orono: Social Science Research Foundation.

Dyer, W. J. (2006). Examining the "Family Effect" on Firm Performance. Family Business Review, 19(4), 253-273.

Eddleston, K. A., Kellermanns. F. W., Sarathy, R. (2008), "Resource configuration in family firms: linking resources, strategic planning and technological opportunities to performance. Journal of Management Studies, 45(1), 26-50.

Fama, E. F., y Jensen, M. C., (1983). Separation of ownership and control. Journal of Law and Economics, 26, 301-325.

Finkelstein, S., y D. C. Hambrick (1996). Strategic leadership: Top executives and their effects on organization. New York: West Publishing Company.

Fletcher, D. (2002). A network perspective of cultural organising and professional management in the small, family business. Journal of Small Business and Enterprise Development, 9(4): 400-15.

Frenz, M., y Ietto-Gillies, G. (2009). The impact on innovation performance of different sources of knowledge: Evidence from the UK Community Innovation Survey. Research Policy, 38(7), 11251135 .
Gallo, M. (1995). The role of family business and its distinctive characteristic behaviour in industrial activity. Family Business Review, 8(2), 83-97.

Gallo, M., Ariño, A., Máñez, I., y Cappuyns K. (2004). La internacionalización a través de alianzas estratégicas en las empresas familiares. Navarra: Universidad de Navarra, IESE.

Galve, C. y Salas, V. (2003). La empresa familiar en España. Fundamentos económicos y resultados. Bilbao: Fundación BBVA.

Gomez-Mejia, L., Nuñez-Nickel, M., y Gutierrez, I. (2001).The role of family ties in agency contracts. Academy of Management Journal, 44, 81-95.

Habbershon, T., y Williams, M. (1999). A resource-based framework for assessing the strategic advantages of famly firms. Family Business Review, $12,1-25$.

Hart, S. (1992). An integrative framework for strategy-making process. Academy of Management Review, 17, 327-331.

Haynes, G.W., Walker, R., Rowe, B.R., y Hong, G.-S. (1999). The intermingling of business and family finances in family-owned businesses. Family Business Review, 12, 225-239.

James, JR., H. (1999). What can the family contribute to business? Examining contractual relationships. Family Business Review, 12, 61-71.

Jantunen, A. (2005). Knowledge-processing capabilities and innovative performance: an empirical study. European Journal of Innovation Management, 8(3), 336-349.

Jensen, M.C., y Meckling, W. (1976). Theory of the firm: managerial behaviour agency costs and ownership structure. Journal of Financial Economics, 3, 305-360.

Kellermanns, F. W. y Eddleston, K. A. (2007). A family perspective on when conflict benefits family firms performance. Journal of Business Research, 60(10), 1048 - 1057.

Kellermanns, F. W., Eddleston, K. A., Barnett, T. y Pearson, A. (2008). An exploratory study of family member characteristics and involvement: Effects on entrepreneurial behavior in the family firm. Family Business Review, 21(1), 1-14.

Kellermanns, F. W., Eddleston, K.A., Sarathy R., y Murphy, F. (2012) Entrepreneurial risk behavior in family firms: A family influence perspective. Small Business Economics Journal, 38(1): 85-101. 
Kirner, E., Kinkel, S., y Jaeger, A. (2009). Innovation paths and the innovation performance of low-technology firms - An empirical analysis of German industry. Research Policy, 38(3), 447-458.

Klomp, L., y Van Leeuwen, G. (2001). Linking innovation and firm performance: A new approach. International Journal of the Economics of Business, 8(3), 343-364.

Kusunoki, K., Nonake, I., y Nagata, A. (1998). Organizational capabilities in product development in Japanese firms: a conceptual framework and empirical findings. Organizational Science, 9(6), 699-718.

Li, H., y Atuahene-Gima, K. (2001). Product innovation strategy and performance of new technology ventures in China. Academy of Management Journal, 44, 1123-1134.

Li, F., y Srinivasan, S. (2011). Corporate governance when founders are directors. Journal of Financial Economics, 102(2), 454-469.

Licht, G., y Zoz, K. (2000). Patents and R\&D: an economic investigation using applications for German, European and US patents by German companies. In: Encaoua, D. et al. (Eds.), The Economics and Econometrics of Innovation. Kluwer Academic publishers, pp. 307-338.

Lokshin, B., Van Gils, A., y Bauer, E. (2009). Crafting firm competencies to improve innovative performance. European Management Journal, 27, 187-196.

Lubatkin M.H., Schulze W.S., Ling Y., y Dino RN (2005). The effects of parental altruism on the governance of family-managed firms. Journal of Organizational Behaviour, 26(3), 313-30.

Marsili, O., y Salter, A. (2006). The Dark Matter of Innovation: Design and Innovative Performance in Dutch Manufacturing. Technology Analysis \& Strategic Management, 18(5), 515.

Miles, R.E., y Snow, C.C. (1978). Organizational strategy, structure, and process. New York: McGraw-Hill Book Co.

Miller D. (1990). Stale in the saddle: CEO tenure and the match between organization and environment. Management Science, 37, 34-52.

Miller D. (1993). The architecture of simplicity. Academy Management Review, 18, 116-138.

Minichilli, A., Corbetta, G., y MacMillan, I.A. (2010). Top Management Teams in FamilyControlled Companies: 'Familiness', 'Faultlines', and Their Impact on Financial Performance. Journal of Management Studies, Special Issue: The Family and Enterprise: Unpacking the Connections, 47(2), 205-222.

Molly, V., Laveren, E., y Deloof, M. (2010). Family Business Succession and Its Impact on Financial Structure and Performance. Family Business Review, 23(2), 131-147.

Muñoz-Bullón F., y Sanchez-Bueno M.J. (2011). The impact of family involvement on the R\&D intensity of publicly traded firms. Family Business Review, 24(1), 62-70.

Mustakallio, M., Autio, E., y Zahra, S.A. (2002). Relational and Contractual Governance in Family Firms: Effects on Strategic Decision Making. Family Business Review, 15(3), 205-222.

Narasimhan, R. y Das, A. (2001). The impact of purchasing integration and practices on manufacturing performance. Journal of Operations Management, 19(5), 593-609.

OECD-EUROSTAT (1997). The measurement of scientific and technological activities. Proposed guidelines for collecting and interpreting technological data, Oslo Manual, OECD, Paris.

Pearson, A., Carr, J. y Shaw J. (2008). Toward a theory of familiness: A social capital perspective. Entrepreneurship Theory and Practice, 32(6), 949969.

Porter, M. (1990). The Competitive Advantage of Nations, Free Press, New York.

Poza, J. E. (2007). Family Businesses, Mason: Thomson South-Western.

Saito, T. (2008). Family firms and firm performance: Evidence from Japan. Journal of the Japanese and International Economies, 22(4), 620646.

Saviotti, P. P., y Metcalfe, J. S. (1984). A theoretical approach to the construction of technological output indicators. Research Policy, 13, 141-151.

Sharma, P. (2008). Commentary: Familiness: Capital stocks and flows between family and business. Entrepreneurship Theory and Practice, 21(6), 971-977.

Sirmon, D. G., y Hitt, M. A. (2003). Managing resources: Linking unique resources, management, and wealth creation in family firms. Entrepreneurship Theory and Practice, 27, 339-358. 
Souitaris, V. (2002). Firm-specific competencies determining technological innovation: a survey in Greece. $R \& D$ Management, 32(1), 61-77.

Steier, L. (2001). Next-generation entrepreneurs and succession: An exploratory study of modes and means of managing social capital. Family Business Review, 14, 259-276.

Tsai, K-H. (2009). Collaborative networks and product innovation performance: Toward a contingenct perspective. Research Policy, 38, 765778 .

Tsai, W.H., Kuo, Y.C., y Hung, J.H. (2009). Corporate diversification and $\mathrm{CEO}$ turnover in family businesses: self-entrenchment or risk reduction? Small Business Economics, 32(1), 57-76.

Valle, S., Avella, L. (2003). Cross-functionallity and leadership of the new product development team. European Journal of Innovation Management, 6(1), 32-47.

Villalonga, B. y Amit, R. (2004). How do family ownership, control, and management affect firm value? WP The Wharton School University of Pennsylvania, pp. 1-34.

Voordeckers, W., Van Gils, A. y Van den Heuvel, J. (2007). Board Composition in Small and Medium Sized Family Firms 2007. Journal of Small Business Management, 45(1), 137-156.

Wagner, J. (2010). International Activities and Firm Performance: Introduction. The World Economy, 33, 311-314

Walters, B.A., Kroll, M.J., y Wright, P. (2007). $\mathrm{CEO}$ tenure, boards of directors, and acquisition performance. Journal of Business Research, 60(4), $331-338$

Wheelwright, S. C., y Clark, K. B. (1992). Revolutionizing product development -quantum leaps in speed, efficiency and quality. The Free Press, New York.

Wiersema M. F., y Bantel K. A. (1992). Top Management Team Demography and Corporate Strategic Change. Academy of Management Journal, 35(1), 91-121.

Wortman, M. S. (1994). Theoretical foundations for family-owned businesses: A conceptual and research based paradigm. Family Business Review, 7(1), 3-27.

Zahra, S. A., y Sharma, P. (2004). Family business Research: a strategic reflection. Family Business Review, 17(4), 331-346.

Zahra, S.A. (2005). Entrepreneurial risk taking in family firms. Family Business Review, 18(1), 23-40.

Zahra, S., Hayton, J., y Salvato, C. (2004). Entrepreneurship in family vs. nonfamily firms: A resource-based analysis of the effect of organizational culture. Entrepreneurship Theory and Practice, 28, 363-381.

Zhan, Q., Doll, W.J. (2001). The fuzzy front end and success of new product development causal model. European Journal of Innovation Management, 4(2), 95-112. 